\title{
Coordination of perception and action in music performance
}

\author{
Peter Q. Pfordresher
}

Department of Psychology and Institute for Music Research, University of Texas at San Antonio

Received 29.06.2005

Accepted 08.10.2005

\section{Keywords}

music performance, sequence production, auditory feedback

\section{ABSTRACT}

This review summarizes recent research on the way in which music performance may rely on the perception of sounds that accompany actions (termed auditory feedback). Alterations of auditory feedback can profoundly disrupt performance, though not all alterations cause disruption and different alterations generate dif- ferent types of disruption. Recent results have revealed a basic distinction between the role of feedback contents (musical pitch) and the degree to which feedback onsets are synchronized with actions. These results further suggest a theoretical framework for the coordination of actions with feedback in which perception and action share a common representation of sequence structure.

\section{INTRODUCTION}

When performing musical sequences, people plan actions to produce acoustic events that communicate thoughts and emotions (for reviews see Gabrielsson, 1999; 2003; Jones \& Holleran, 1992; Juslin \& Sloboda, 2001; Palmer, 1997; Sloboda, 1982; 1985; 2005). Sequence production in contexts such as music and speech involves communicating a message through sound, a process that is probably guided by monitoring the perceived consequences of one's actions (Levelt, 1989; MacKay, 1987; Palmer \& Drake, 1997). However, little research has addressed the role of self-perception in music performance. My research has explored this issue by examining the ways in which the maintenance of fluency during music performance depends on matches between actions and auditory feedback (the sounds one creates). Results suggest that higher-order representations of musical structure guide both the planning of actions and the perception of auditory feedback (cf. Hommel, Müsseler, Aschersleben, \& Prinz, 2001; MacKay, 1987; Müsseler, 1999; Prinz, 1997).

The lack of research on the role of auditory feedback may result from its apparently obvious importance to music performance. How else could music be learned or communicated but through close monitoring of sound? Nevertheless, some evidence indicates that the importance of auditory feedback may be circumscribed. On the one hand, the presence of sound during learning facilitates recall during performance, even for skilled pianists (Finney \& Palmer, 2003). On the other hand, learning to produce a sequence of key presses on a piano does not depend on the presence of sound, even for people without any musical training (Pfordresher, 2005, Experiment 3). Furthermore, the absence of sound during keyboard performance of a learned melody has negligible effects on trained performers (Finney, 1997; Finney \& Palmer, 2003; Gates \& Bradshaw, 1974; Pfordresher, 2005; Repp, 1999; Repp \& Knoblich, 2004) or untrained performers (Pfordresher, 2005).

In contrast to null effects of feedback absence, certain alterations of auditory feedback can profoundly debilitate performance, to the extent that a skilled performer sounds like a beginner. Thus, pianists may

Correspondence address: Peter Q. Pfordresher, Ph.D., Dept. of Psychology and Institute for Music Research, University of Texas at San Antonio, 6900 North Loop 1604 West, San Antonio, TX 78249, email: Peter.Pfordresher@utsa.edu 
rely on certain matches between auditory feedback and actions when auditory feedback is present, though they may not rely on the presence of feedback. Disruption from altered feedback therefore may result from miscoordination between perception and action. The present article reviews research on the disruptive effects of altered auditory feedback to music performance, focusing in particular on the effects by the author (for more extensive reviews on the role of auditory feedback see Finney, 1999; Smith, 1962; Yates, 1963; for reviews focused on speech with applications for stuttering see Howell, 2004a, 2004b). The focus of the research summarized here is on keyboard performance, which is the only domain of music performance that has been studied extensively with respect to altered auditory feedback. This review is organized into two main sections, the first summarizes recent results of different kinds of feedback alterations, and the second reflects on the theoretical implications of these results.

\section{EFFECTS OF ALTERED AUDITORY FEEDBACK (AAF)}

Research on the interplay between perception and action in music performance has relied mainly on the altered auditory feedback (AAF) paradigm, in which each sound that results from a produced action (e.g., a key press) is altered. The result of any alteration is that the coordination of auditory feedback with actions is changed. The two primary types of alterations occur along the dimensions of pitch and time. In the present context, "time" will refer specifically to onset synchrony (i.e., whether a sound occurs at the time a key is pressed), whereas changes exclusive to pitch occur when sounds are synchronized with key presses but pitches deviate from what one would normally hear on a keyboard. Combined alterations occur when sounds are both asynchronous and changed in pitch.

\section{Manipulations of feedback timing (synchrony)}

The most extensively studied feedback alteration concerns timing (synchrony) of perception and action: Delayed Auditory Feedback (DAF, a specific type of AAF). In this paradigm a constant time lag is inserted between produced actions and the onsets of auditory feedback events, such that the onset of a feedback event (in music, a single pitch or chord) from an action produced at time $t$ occurs at time $t+d$. Black (1951) and Lee (1950) independently discovered the disruptive effect of DAF on speech produc- tion by having participants hear auditory feedback that was delayed via a tape loop. Lee (1951) observed that speech with DAF sounded like stuttered speech (but see Howell, 2004b): Speakers slowed down, prolonged vowels, and generated more dysfluencies when speaking with delayed auditory feedback compared to speech with normal feedback. Research on the effect of DAF on music performance began with Havlicek (1968; though reference to "preliminary experiments" was made by Kalmus, Denes, \& Fry, 1955), who showed that DAF disrupted performance on many musical instruments, including keyboard, brass, woodwind, and string instruments.

Recent research supports two general conclusions about the role of feedback timing: (a) Asynchronies between actions and feedback primarily disrupt the timing of actions, not their sequencing, and (b) the degree of disruption that results from asynchronies primarily reflects phase relationships between the onsets of feedback sounds relative to produced time intervals between key presses rather than the absolute time discrepancy.

\section{Feedback timing and type of disruption}

As mentioned before, DAF influences many characteristics of performance. Similar to effects on speech, DAF disrupts keyboard performance by increasing error rates (e.g., Finney, 1997), increasing inter-onset intervals (IOIs, e.g., Gates, Bradshaw \& Nettleton, 1974; Havliceck, 1968; Finney, 1997), and increasing timing variability (Pfordresher, 2003a; Pfordresher \& Palmer, 2002). Increases in each variable are evidence of "disruption" insofar as they signal a deviation from the intended performance, typically represented by performance with normal feedback. Note that increases in timing variability can only be considered evidence of disruption for expressively "flat" (mechanical) perfo rmances of isochronous melodies; otherwise increased timing variability could result from changes to expressive nuances brought about by altered feedback.

Pfordresher (2003a) proposed that the diverse types of disruption found in earlier work may reflect the fact that relationships between DAF onsets and produced actions vary with tempo. This point is illustrated in Figure 1. When IOIs are longer than delays (Figure 1, top), DAF disruption would seem to result purely from asynchronies. The anticipated feedback pitch follows after its associated action but before the next action. However, when IOIs are equal to delays (Figure 1, middle) one could experience synchronous feedback that differs with respect to the expected pitch. In such cases disruption would result solely from deviations of feed- 

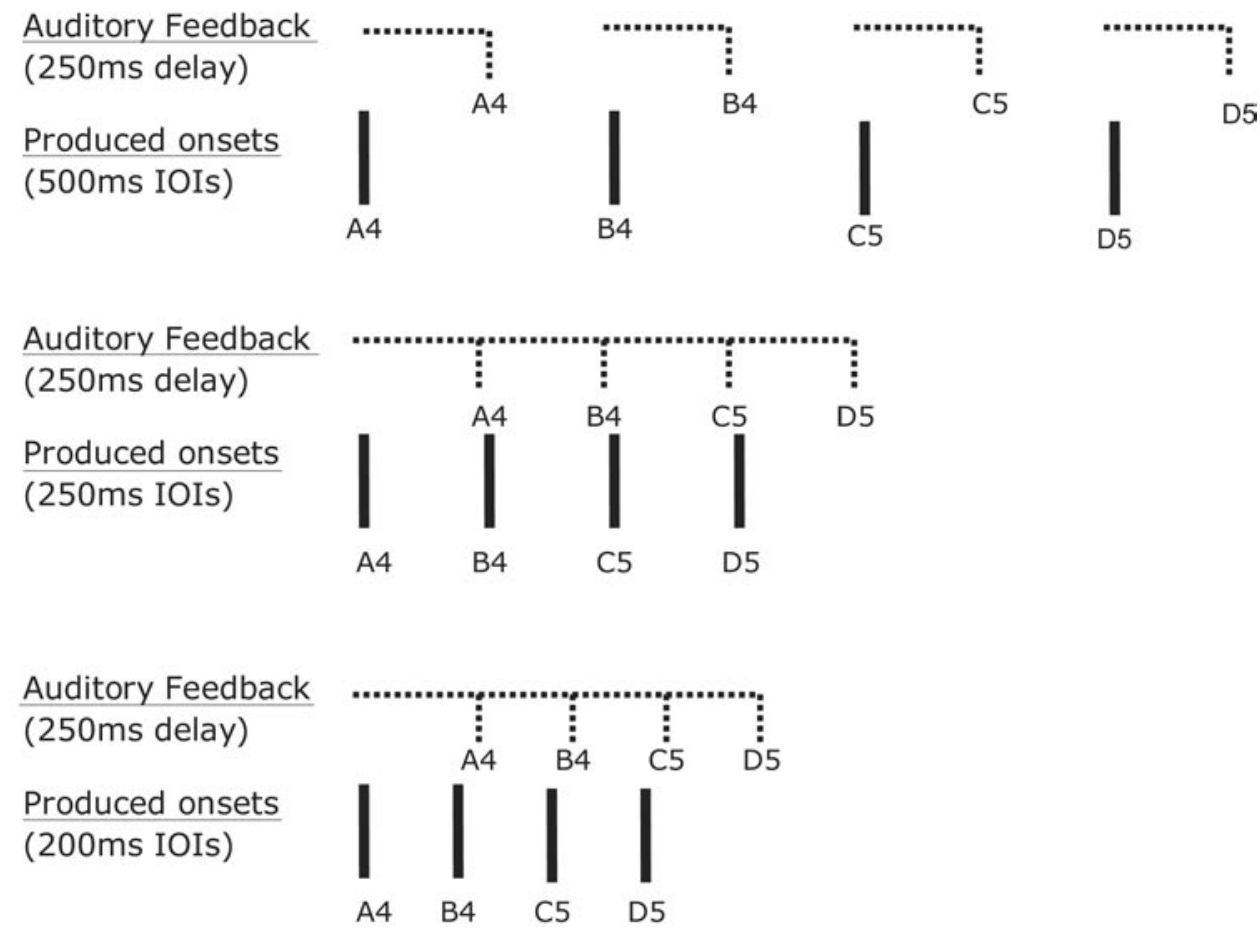

\section{Figure 1.}

Relationships between produced actions (solid lines) and auditory feedback onsets (dotted lines) as a function of production rate (tempo) in a typical DAF paradigm. Vertical lines indicate produced (solid) and perceived (dotted) event onsets. Letternumber combinations indicate musical pitch class and octave, respectively.

back from the expected pitches. Finally, when IOIs are shorter than delays (Figure 1, bottom), feedback onsets both lag behind produced actions and also present an unexpected pitch. Disruption in such circumstances may reflect both feedback timing and pitch.

Alterations of feedback timing primarily disrupt produced timing when feedback event onsets are asynchronous with produced event $i$ but precede produced event $i+1$. This was demonstrated unambiguously by Pfordresher (2003a, Experiment 1; see also Pfordresher $\&$ Benitez, 2006). In that experiment, a new kind of delay was used that adjusted to produced timing such that feedback onsets maintained a roughly consistent relative phase position within produced IOIs (manipulated using FTAP, Finney, 2001). Whereas these adjustable delays (called "phase shifts") increased timing variability and slowed production rate, they only marginally increased error rates relative to trials with normal feedback. Conversely, other experiments in the same study (Pfordresher, 2003a, Experiments 2 and 4) demonstrated that alterations of feedback pitch without asynchrony increased errors but did not influence produced timing.

This dissociation suggests that the nature of the perception/action relationship that is changed in AAF determines the aspect of production that is disrupted.
Thus, timing of performance depends on timing of auditory feedback, and pitch accuracy in performance depends on the pitch structure of auditory feedback, a point that will be elaborated on later.

\section{Feedback timing and maximal disruption}

A major issue in research on delayed auditory feedback has been the amount of delay that causes maximal disruption. Typically, disruption increases with delay length (temporal separation between actions and feedback) to a certain point and then reaches asymptote (e.g., Gates, Bradshaw, \& Nettleton, 1974, who found an asymptote around $270 \mathrm{~ms}$ in music performance) or decreases (e.g., in speech, Fairbanks \& Guttman, 1958). Some speech researchers have proposed that maximal disruption occurs when delay lengths equal the length of the syllable (approximately $200 \mathrm{~ms}$ at a normal speakeing rate) - a possible planning unit in speech (Black, 1951; see also Howell, Powell, \& Khan, 1983). This claim is supported by the finding that the delay causing maximal disruption is greater (longer) when people speak at a slower rate (Robinson, 1972).

An alternative proposal is that the delay causing maximal disruption reflects a "critical interval" between actions and feedback that is independent of 
production rate (e.g., Chase, Harvey, Standfast, Rapin, \& Sutton, 1961; MacKay, 1987). The node structure theory of Mackay (1987) offers an account of AAF disruption based on the assumption of a critical interval. The theory proposes that perception and action planning use a common set of hierarchically organized content nodes. An action occurs when a content node is activated. Following activation, the content node enters a hyper-sensitive phase, at which point DAF onsets can re-trigger the action, leading to repetition errors. This hyper-sensitive phase is hypothesized to peak around $200 \mathrm{~ms}$. In support of node structure theory (and in contrast to Robinson, 1972), some researchers have failed to find an influence of production rate on the maximally disruptive delay for spoken sequences produced at different rates (Butler \& Galloway, 1957; Mackay, 1968). It is possible, however, that the discrepancy between these results and those of Robinson (1972) was found because Robinson controlled speaking rate by having subjects synchronize syllable onsets with a metronome, whereas the other studies had subjects speak at subjectively "fast" or "slow" rates.

A broader implication of these claims is that disruption may be a function of either the relative phase relationship between the timing of actions and feedback onsets, or of the absolute temporal separation between an action and its associated feedback event. The relative phase view predicts that, disruption will scale with the position of auditory feedback onsets relative to the recurring cycle formed by produced IOIs, regardless of production rate (tempo). Normal feedback represents phase synchrony (e.g., for no delay, phase $=0$ ). Increased delay lengths within a tempo condition lead to increased phase ratios until the point at which delays equal IOIs, when phase synchrony is regained (delay phase $=1$ ). When the IOIs are variable, a fixed feedback delay produces variable phase ratios across the sequence. To achieve fixed phase delays, the feedback delay must be made contingent on IOI duration. This is difficult to achieve when IOIs are formed by onsets of spoken syllables, which are highly variable (e.g. Lehiste, 1977), but easier to achieve in the performance of isochronous melodies, where deviations from isochrony are relatively small.

Recent work in music performance, which has examined the influences of delay phase and delay length independently, generally supports the idea that relative phase (i.e., rhythmic) relationships between actions and feedback determine the amount of disruption that results. Recent research has compared the influence of fixed time delays (like traditional DAF) and adjustable delays for performances at three different tempi, and has also demonstrated that relative phase provides the best account of increasing disruption (Pfordresher \& Benitez, 2006), regardless of whether delays maintained fixed durations or varied in order to maintain constant relative phase. Earlier work supports this result. Pfordresher \& Palmer (2002) found that the variability of produced timing (an index of timing disruption) increased with delay beyond the $200 \mathrm{~ms}$ interval shown to be maximally disruptive in other research (MacKay, 1987). Disruption increased as feedback onsets approached the time of the next action (cf. Finney \& Warren, 2002), regardless of tempo. These findings were confirmed in research with phase shifts described earlier (Pfordresher, 2003a, Experiment 1). Thus it is possible that findings supporting the notion of an absolute critical interval may result from poor control of timing relationships, given that those studies examined speech produced at qualitatively defined rates.

\section{The influence of simple versus complex phase ratios}

Certain phase relationships appear to be favored in coordinative behavior. For instance, coordination of two limbs that execute repetitive rhythmic movements (e.g., swinging the arms) is more accurate and precise when each limb's cycle is either in phase (a 1.0 phase ratio) or anti-phase (0.5), relative to the other limb's cycle. Typically, phase synchrony exhibits more stable coordination than anti-phase coordination (for reviews see Kelso, 1995; Rosenbaum, 1991; Schmidt \& Lee, 1999; Turvey, 1990). Similar results have been found for perceptual behaviors. For instance, subjects can track fluctuations in the movements of two oscillating dots across a screen more accurately if the two movement patterns are coordinated in phase or anti-phase than for other regimes (Bingham, Schmidt \& Zaal, 1999; Zaal, Bingham \& Schmidt, 2000).

Given that coordination within each modality (action versus perception) favors simple phase relationships, it is plausible that coordination of perception with action will also favor simple phase relationships. In the context of delayed feedback, this view predicts a decrease in disruption for phase synchrony (delayed feedback coinciding with key presses) or alternation (feedback onsets occurring in between key presses.

In support of this prediction, the disruption of timing with increasing phase of delayed feedback has been found to drop abruptly when the delays are equal to the produced IOIs, conforming to a simple (1.0) phase 
ratio (Pfordresher, 2003a; Pfordresher \& Benitez, 2006). Of course, during performance of melodies on the piano, such phase lags result in participants hearing the previously produced pitch when they are depressing each key, which may increase disruption of accuracy (though not timing), an issue that will be addressed in the next section.

Mixed support has been found for the related prediction that disruption will be reduced when delays fall halfway between produced onsets (a phase ratio of .5). Pfordresher \& Palmer (2002) found reduced timing variability in one of two tempo conditions, relative to the general increase with phase mentioned before. Furthermore, when allowed to choose their own tempo during fixed-delay feedback, participants tended to choose a tempo at which the delays occurred at .5 phase ratios (Experiment 2). The relative advantage for .5 phase ratios has not been replicated in other experiments, however (Pfordresher, 2003a; Pfordresher \& Benitez, 2006).

An apparent exception to the finding that disruption drops for delays forming 1.0 phase ratios was found by Finney and Warren (2002). In that study, participants tapped a rhythmically varying sequence in which a group of 4 isochronous taps was followed by a group of 2 taps, with pauses between groups being equal to two withingroup IOIs (cf. Kalmus et al., 1955). Thus, short IOIs formed a referent beat and the generation of a pause in production involved skipping a beat. Because the procedure involved tapping monotone sequences, deviations of feedback pitch from the expected pitch could not influence the results. The authors found maximal disruption when delay lengths were equal to the within-group IOI. Importantly, disruption primarily took the form of insertion errors (but see Finney, 1999 for discussion of smaller effects on timing). The authors interpreted this finding as a form of rhythmic entrainment (cf. Large \& Jones, 1999). Specifically, when the rhythmic pattern formed by auditory feedback is phase-shifted relative to the produced rhythmic pattern, the performer inserts an additional action to regain synchrony between the two patterns. Thus, despite seemingly different results, the reason behind disruption (of accuracy) found by Finney and Warren reflects the same underlying tendency to synchronize actions with feedback that resulted in decreased disruption (of timing) by Pfordresher (2003a; Pfordresher \& Benitez, 2006).

Taken together, these results support the idea that asynchronous feedback disrupts produced timing because of relative timing (i.e., rhythmic) relationships between perception and actions, and that less disruption occurs when these relationships form simple phase ratios (1.0, and perhaps 0.5). Howell et al. (1983) made a similar proposal in the context of speech but limited disruptive rhythms to those in which the amplitude peak of auditory feedback coincides with the offset of a produced action. Howell et al. (see also Howell \& Archer, 1984) made a further claim that will be addressed in the next section: that AAF disruption is limited to onset asynchrony, and does not occur when changes are specific to the contents of auditory feedback (e.g., pitch).

\section{Manipulations of feedback contents}

In contrast to timing of auditory feedback, the influence of feedback content is less well explored. Feedback content refers to the auditory event category (e.g., musical pitch) that results from a produced action. Manipulations of feedback contents during musical keyboard performance involve altering the relationships between piano keys and resulting pitches such that an unexpected pitch sounds in synchrony with each key press during a performance. Such manipulations are theoretically important because they create mismatches between feedback and the planned consequences of actions without disrupting their synchrony (cf. Howell, 2004b).

Some early work suggested that alterations of feedback content have little influence on the fluency of music or speech production. For instance, Howell and Archer (1984) compared the timing of speech with typical DAF to a condition in which delayed feedback was further transformed to a continuous $500 \mathrm{~Hz}$ squarewave tone. The amplitude contour of the speech was preserved but the phonemic content and pitch contour were removed from auditory feedback. Howell and Archer found no differences between these conditions and concluded that feedback timing alone influences production (but see Mackay, 1987, for a discussion of contrasting findings). Similarly, Finney (1997) manipulated feedback pitches during piano performance in various ways, all of which involved mapping piano keys to alternate pitches. These alterations did not disrupt performance, in contrast to significant disruption (with respect to timing as well as errors) from a typical DAF condition. Note that these manipulations of feedback content resulted in a sequence of events that was highly dissimilar to the planned sequence of events and therefore may have been perceived as being unrelated to planned actions.

Recent research has shown that disruption can occur when feedback contents are altered in a way that results in a feedback pitch sequence that resem- 
Performed :

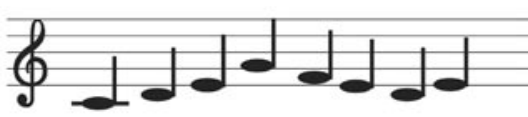

Feedback :

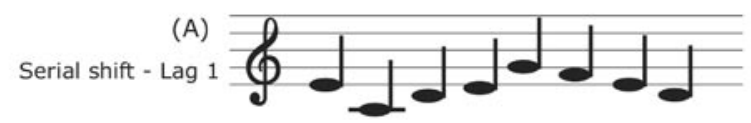

(B)

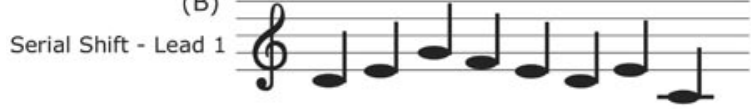

(C)

Permuted Order

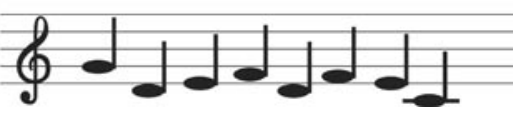

Figure 2.

Relationships between the planned sequence of pitches (actions), shown in the top stave, and the resulting sequence of auditory feedback pitches in different altered auditory feedback conditions $(A-C)$

bles but is not identical to the planned pitch sequence. Compared to asynchronous feedback, alterations of contents reliably increase error rates but have smaller effects on timing variability and negligible effects on production rate (Pfordresher, 2003a). Thus, alterations of content may disrupt a process that regulates serial order rather than timing.

\section{The disruptive effect of serial shifts}

Robust disruption has been found when participants hear feedback that is serially shifted with respect to produced sequences. ${ }^{1}$ Serially shifted feedback causes the feedback event at each key press to match a pitch intended for a different sequence position, with a constant serial separation between the current position and the position associated with the feedback pitch. For instance, a lag-1 serial shift causes the performer to hear the pitch associated with the previous key press. For example, Figure $2 \mathrm{~A}$ shows the lag- 1 feedback sequence one would hear while producing the melody represented by the notation at the top of Figure 2. Disruptive effects of serial shifts from past (Pfordresher, 2003a, 2005; Pfordresher \& Palmer, in press) and future (Pfordresher \& Palmer, in press, see Figure 2B) events have been documented, for separations of $+/-1$ to 3 events. Overall disruption was not influenced by the amount of serial lag or lead or by whether feedback events originated from future or past events.

The serial separation between the current position in a performance and the shifted feedback may nevertheless influence the kinds of errors that occur. Pfordresher and Palmer (in press) analyzed the influ- ence of serial lags and leads on serial ordering errors: errors involving the production of an event intended for a different sequence position (cf. Dell, 1986; Fromkin, 1971; Garrett, 1980; Lashley, 1951). Serial ordering error frequencies were analyzed with respect to direction (anticipations versus perseverations) as well as with respect to distance (how far away the intended position of the error is relative to the current position). We had hypothesized that serial shifts would increase the production of events at the distance/direction that matches the relationship between actions and auditory feedback (e.g., that "feedback" from 2 events ahead would increase anticipatory errors from 2 events away). Such results were not found, however. Instead, patterns of serial ordering errors suggested reverse adaptation, such that perseveratory errors increased in the presence of feedback from future events and vice-versa.

\section{Feedback contents and structural similarity}

Arguably, serial shifts could be said to influence perception/action coordination in the same way as DAF, only on a different time scale. In other words, lag-1 serial shifts can be considered a type of delay that is synchronous rather than asynchronous, and the resulting disruption may reflect some kind of response to temporal separation of actions from feedback. Results thus far argue against such an interpretation. As said earlier, serial shifts disrupt different aspects of production than do asynchronous delays. Furthermore, the degree of serial separation does not modulate disruption, whereas the phase position of asynchronous delays does influence the magnitude of disruption. Additional evidence bolsters the distinction between alterations of synchrony versus contents by demonstrating that alterations of pitch other than serial shifts can disrupt performance. Importantly, alterations of pitch contents that cause disruption do so when the feedback sequence is structurally similar to the planned sequence with respect to the pattern of pitch motion and tonal structure.

Pfordresher (2005) compared the disruptive effects of lag- 1 serial shifts to those of other alterations that generated feedback sequences of varying structural similarity to the planned sequence. Although lag-1 shifts consistently elicited the highest disruption, significant disruption was also found for a condition in which the feedback sequence comprised a random permutation of pitches from the planned sequence (see Figure 2C). By contrast, sequences comprising randomly ordered pitches that did not match any pitch in the planned sequence elicited negligible disrup- 
tion, regardless of whether these pitches fell within the same pitch range as the produced melody or were drawn from a broader pitch range.

Further support for the idea that disruption from lag1 serial shifts reflects structural similarity stems from recent data (Pfordresher, 2006). In that study, feedback melodies could be transposed to a different key (e.g., from C major to F\# major), or could be transformed to "contour foils" that maintain the planned melody's pattern of pitch change with respect to direction but not extent (i.e., the size of pitch intervals). Pianists showed similar disruption when a transposed melody or a tonal contour foil was serially shifted as they did during a typical lag-1 serial shift, although the same effect was not found for atonal contour foils (in which constituent pitches were drawn from the chromatic scale, cf. Dowling, 1978). Importantly, serial shifts of transposed melodies or tonal contour foils cannot be interpreted as being similar to delayed feedback, because pitches of individual events in the feedback sequence do not match any single pitch in from the planned sequence in absolute respects. Instead the serial shifting pertains only to the pattern of pitch motion relative to movements. Other preliminary data indicate that presenting feedback sequences with a reversed melodic contour, relative to the planned sequence, causes amounts of disruption similar to those caused by serial shifts. Thus, the pattern of pitch changes in feedback sequences relative to the pattern of finger movements may cause disruption, rather than serial displacement between actions and associated feedback. Furthermore, the fact that serial shifts of atonal contour foils do not cause disruption suggests that performers are sensitive to similarity between planned and feedback sequences at the level of tonal schemata as well.

One component of similarity in musical structure is meter, the alternating strong and weak beats that help to characterize a melody's temporal structure (e.g., Cooper \& Meyer, 1960; Lerdahl \& Jackendoff, 1983; Palmer \& Krumhansl, 1990; Palmer \& Pfordresher, 2003). Pfordresher (2003a) reported results suggesting that performance with altered feedback contents is facilitated (i.e., less disrupted) when the pattern of metrical accents in auditory feedback matches the pattern of metrical accents associated with planned events. Reduced disruption was found for serial shifts of lag 2 during performances of melodies with a 2/4 (binary) meter, relative to serial shifts of lags 1 or 3 . Likewise, reduced disruption was found for lags of 3 during performances of melodies with a 3/4 (ternary) meter, relative to lags of 2 (but not 1 ). This result was highly qualified by experience, however. Only pianists with an intermediate level of training showed facilitation from matching metrical accents. Highly skilled pianists, who tended to make fewer errors, as well as unskilled pianists, who made many errors, showed similar disruption across all lags and performed meters.

\section{The role of musical skill}

It is possible that disruption from altered feedback contents depends on the acquisition of musical skill, given that disruption from altered contents must result from the fact that perceived pitches differ form those one would usually hear on a piano. Associations between keys and sounds are strengthened during skill acquisition (Bangert \& Altenmüller, 2003; Drost, Rieger, Brass, Gunter, \& Prinz, 2005). Based on this evidence, one might not expect any influence of altered feedback contents when an untrained individual played a melody for the first time on a piano. Moreover, given that musical schemata, such as tonality, are enhanced via training (e.g., Krumhansl \& Shepard, 1979) it is possible that non-pianists would not respond to global similarity relationships between feedback sequences and planned sequences.

Recent research has compared responses of trained pianists and individuals with little or no formal piano training to the same auditory feedback conditions. Tasks have typically been constructed differently for both groups in order to equate difficulty (as opposed to equating the specific task conditions). Whereas pianists perform longer more complex melodies from music notation, non-pianists perform melodies using a numerical coding system based on specific finger-key relationships for the right hand (see Pfordresher, 2005, for details). These tasks have been shown to elicit similar overall error rates for the different groups, although non-pianists were much more variable in terms of produced timing than were pianists.

Contrary to intuition, pianists and non-pianists responded similarly to alterations of feedback contents (Pfordresher, 2005). These results were not dependent on the presence of sound during learning of the melody (Experiment 5). Furthermore, non-pianists were able to discriminate the melody they performed from alternative melodies (Experiment 6). More recent data have also demonstrated that the aforementioned dissociation between the effects of alterations to feedback contents versus timing holds for non-pianists (Benitez, 2005). Thus, disruption from altered feedback contents may reflect general sensitivity to correlations between patterns of planned movements and patterns of pitch motion. More recent evidence 
suggests some limitations to similarities across experience groups. Pianists show negligible disruption when the feedback melody is simply transposed such that each feedback pitch is 6 semitones higher than the expected pitch. Conversely, non-pianists do show some disruption from this manipulation, probably resulting from the fact that non-pianists have not learned to treat transpositions as identical to the planned melody (Pfordresher, 2006).

An issue related to skill acquisition is the possibility that performers may adapt to AAF during an experimental session. That is, performing with AAF may constitute a skill that participants acquire, similar to the way in which many pipe organ players must adapt to feedback delays that can occur when using that instrument. Early research on DAF indicates minimal adaptation to those alterations within an experimental session (Yates, 1963, pp. 219-220). Results may differ, of course, for alterations of feedback content. In the domain of speech, Houde and Jordan (1998) did find adaptation to AAF when feedback from produced vowels was altered to match the sounds of other vowels; speakers adapted production to maintain the sound of the target vowel. In addition, Houde and Jordan found after-effects when feedback was changed back to normal, as well as generalization of after-effects to vowels that were not produced with AAF. Research on music performance has not been designed to test practice or adaptation effects. Nevertheless, changes in patterns of serial ordering errors for serial shifts from the past and future, discussed earlier, suggest compensation of planning mechanisms in the presence of AAF (Pfordresher \& Palmer, in press). Thus far my own data do not reveal reliable effects of practice, according to post-hoc analyses I conducted for the purposes of this review. However, as mentioned earlier, these experiments were not designed to test practice effects. Thus the nature of adaptation to AAF, especially over the long term (as in the case of organ players) is still open to investigation.

\section{Combined manipulations of contents and synchrony}

Altered auditory feedback can be both asynchronous and different from the expected pitch (e.g., resulting from the previous key press). Such conditions can be considered as combined alterations of feedback timing and feedback contents.

An intuitive prediction regarding combined alterations is that they might reflect the summed effects of asynchrony and changed contents, resulting in ro- bust disruption of both produced timing and accuracy. Existing evidence indicates the contrary. Both Finney (1997) and Pfordresher (2003a) found significant reductions in disruption for combined alterations. Finney combined DAF delays of 200 ms with scrambled feedback pitch, whereas Pfordresher combined phase shifts with serially shifted feedback. Moreover, although Pfordresher's manipulations could be considered as variations of overall delay (e.g. a serial shift of lag 1 plus a phase shift of $50 \%$ is a $150 \%$ phase shift), patterns of disruption across "time" construed this way did not reflect a simple function of time. A likely explanation for these findings is that combined delays cause the feedback sequence to be perceived as unrelated to the planned sequence. Thus, the AAF paradigm may pose a "binding" problem between perception and action with respect to the relationship between perceived events and actions (cf. Singer, 1993).

Both these findings contrast with Howell and Archer (1984), who found that combining DAF with an alteration of contents that converted speech to square wave tones yielded the same disruption as DAF alone. It is possible that the discrepancy between these results reflects the nature of the alteration to feedback contents. Whereas the manipulations of Pfordresher and Finney created a contrasting pattern of pitch motion, the manipulation of Howell and Archer resulted in a monotone sequence that may have been easier to ignore.

\section{Individual differences}

It has long been noted that striking differences exist with respect to how much disruption an individual experiences from DAF (e.g., Yates, 1963). Sources of individual variability have been hard to identify, however, and some researchers have suggested that these differences may simply reflect different strategies to overcome disruption (Howell \& Archer, 1984).

Recent work has explored three theoretically-motivated factors that might account for individual differences (Benitez, 2005): Music perception/memory ability, general intelligence, and the personality facet of deliberation (from the "Big 5" inventory, Costa \& McCrae, 1992; Piedmont, 1998). Music perception/memory ability was measured with the Montreal Battery of Evaluation of Amusia (Peretz, Champod, \& Hyde, 2003), in which listeners make same/different judgments about pairs of melodies that differ with respect to the pitch or timing of one event. It was reasoned that sensitivity to melodic structure may influence sensitivity to relationships between planned and 
perceived sounds. Measures of general intelligence (using the Wonderlic Personnel inventory, Wonderlic, 1992) and deliberation were used to gauge whether differences in planning abilities modulate disruption from AAF. An enhanced ability to process information (high general intelligence) or a tendency to take the appropriate time to form plans in advance of production (the theoretical basis for deliberation) may result in action plans that are resistant to AAF disruption.

Benitez (2005) tested the degree to which each of these measures predict the degree of disruption individuals experience from phase shifted or serially shifted auditory feedback. The sample included 101 individuals, most of whom had no formal musical training. Results supported the idea that people high on deliberation experience less disruption of accuracy from serial shifts than do people who are low on deliberation. Deliberation did not predict disruption of timing from asynchronous delays, however. In addition, poor performance on the rhythmic subtest of the MBEA predicted greater disruption of timing from asynchronous delays, but not disruption from serial shifts (performance on pitch related tasks on the MBEA did not predict levels of disruption). Thus, individuals whose personalities are characterized in general by deliberation may develop more well-formed sequential plans that are less prone to disruption, and individuals who are better at perceiving and remembering rhythmic relations in music may be better at maintaining temporal regularity in the presence of interfering sounds. General intelligence did not predict the degree of disruption, despite recent evidence suggesting that general intelligence and musical performance skill may be linked (e.g., Rauscher et al., 1997; Schellenberg, 2004; but see Costa-Giomi, 1999, 2004)

\section{THEORETICAL IMPLICATIONS}

These results do not conform to any single existing theory that addresses the use of auditory feedback. I propose a theoretical framework that combines assumptions from earlier approaches, based on the idea that perception and action share a common representation of sequence structure in memory (cf. Hommel et al., 2001; MacKay, 1987; Müsseler, 1999; Prinz, 1997).

\section{Comparison to earlier theories}

Clearly, disruption is not limited to asynchronous AAF. Thus, the rhythmic displacement hypothesis, which limits disruption to such circumstances, is too narrow (Howell et al., 1983). More recent modifications of this hypothesis have suggested that "additional" synchronous feedback, such as the sound of another person speaking while you speak, can slow production (Howell, 2001, 2004b). However, the evidence summarized here shows that AAF can influence production when it is neither synchronous nor presented in addition to correct feedback.

Disruption from AAF does seem to be related to rhythmic relationships between actions and feedback, albeit not limited in the way proposed by Howell and colleagues. Take for instance the fact that serial shifts result in contrasts between the melodic contour of the feedback sequence and the planned pattern of movements (Pfordresher, 2006). Such alterations can be considered a kind of disruptive rhythm based on the fact that melodic contour creates accents that attract attention and influence the perception of rhythm (e.g., Boltz \& Jones, 1986; Jones, 1987; Jones \& Pfordresher, 1997; Pfordresher, 2003b; Thomassen, 1982). Thus, the role of rhythm in perception/action coordination may extend beyond onset synchrony to incorporate structural characteristics. This idea follows from recent research and theory suggesting that temporal coordination results from the cognitive representation of time rather than emergent properties of muscle movements (see Ivry, Diedrichsen, Spencer, Hazeltine, \& Semjen, 2004, for a review).

Traditional feedback control hypotheses have trouble accounting for recent results. Feedback control theories share an emphasis on the use of feedback for error correction (e.g., Chase, 1965). Thus, any kind of alteration to auditory feedback should signal that an error has occurred, leading to disruption. Related models have proposed that auditory feedback triggers a serial chaining mechanism (e.g., Fairbanks \& Guttman, 1958; Lee, 1950), and that DAF disrupts the timing of this mechanism. By this account, the absence of feedback should result in the inability to retrieve sequence events. In contrast to these predictions, not all alterations of pitch contents disrupt production, and removal of feedback yields negligible effects on production.

Although feedback control theories propose a link between perception and action that is too limited, the notion that feedback is related to plans for actions seems warranted. The shortcomings of feedback control theories result from a limited conceptualization of what constitutes a plan. These theories have focused on relationships between feedback and planning that are limited to individual events (e.g., the specific pitch that results from a single key press on the piano). It is likely, however, that relationships between feedback 
Shared Representation

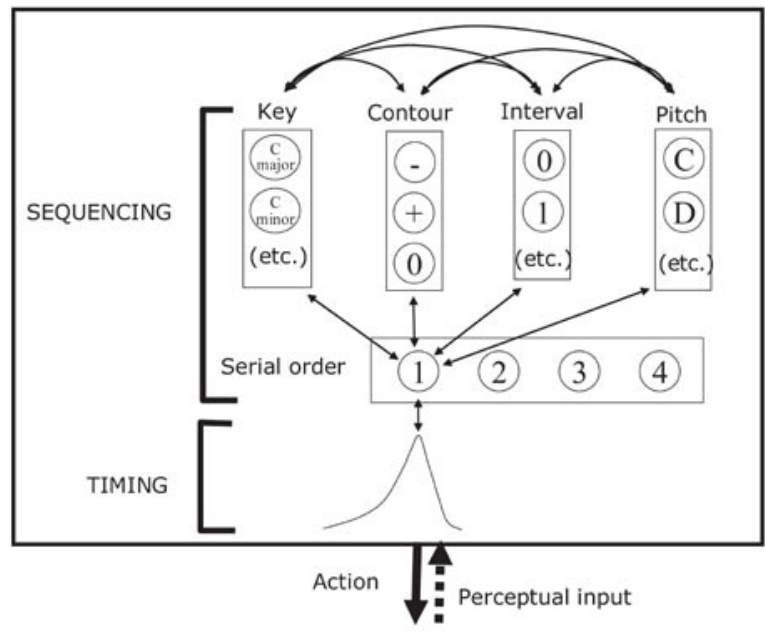

Figure 3.

The shared representation framework. Components inside the box make up the plan that guides both action and perception. Two-headed arrows indicate bi-directional links. For simplicity, not all possible connections are shown

and planning incorporate sequential relationships across a series of actions and their relationship with a concurrent feedback sequence. In addition, feedback control accounts may adopt an overly passive view of how people respond to auditory feedback, in light of recent evidence suggesting that performers may adapt planning mechanisms in the presence of AAF (Pfordresher \& Palmer, in press).

Finally, various findings run counter to the idea that a single delay length generates maximal disruption under a variety of circumstances, a claim developed most thoroughly in Node Structure Theory (MacKay, 1987). Although the assumption of a critical interval proposed by Mackay (1987) seems untenable, the general notion that AAF disrupts production by adding activation to events planned for other locations is plausible. Thus, in the following sections I outline the basic components of a theory of perception and action that explains the influence of AAF on music performance by integrating aspects of existing theories.

\section{Preliminaries to a new theory}

At this stage, no formal model exists that can relate auditory feedback to performance, although a close approximation is the model of Mackay (1987, though it has not been instantiated to my knowledge). A schematic outline of a new theoretical framework is presented in Figure 3. The model assumes a basic functional separation between sequencing and timing (Pfordresher, 2003a; see also Krampe, Mayr, \& Kliegl, 2005; MacKay, 1987), as well as the idea that representation of musical structure exists at multiple time scales (Jones, 1976; Jones \& Boltz, 1989). Different components of sequential structure are shown as different components of a connectionist network (not all connections are shown for sake of simplicity). Components are limited to those that are important for the coordination of perception and action according to the summarized research.

\section{Perception and action share a common representation}

The most fundamental assumption of the proposed framework is that a common shared representation of sequence structure in memory guides planning of actions and interpreting the perceived consequences of those actions. This shared representation view (Hommel et al., 2001; MacKay, 1987; Müsseler, 1999; Prinz, 1997) is consistent with recent neurophysiological evidence for "mirror neurons" that respond similarly when a monkey or human executes and action or observes another individual executing the same action (Rizzolatti, Fogassi \& Gallese, 2001). By this account, humans use the same conceptualization of an event sequence to retrieve events for action and to perceive the consequences of those actions. This proposal is also similar to ideo-motor theory, in that it suggests that action planning is linked to anticipations of the perceptual outcomes of those actions (Greenwald, 1970; James, 1890).

The representation of a sequence must distinguish events by their serial position. Thus the accessibility of sequence events at different positions varies over time, with the current event being most accessible. Accessibility is commonly assumed to be governed by levels of activation in nodes that represent different dimensions of event content (e.g., Dell, 1986; cf. the role of priming in MacKay, 1987). With respect to production, activation reflects the likelihood that an event is retrieved and produced (cf. MacKay, 1987). With respect to perception, activation reflects the match between perceptual input and a represented event.

In the proposed framework, event accessibility during production is determined jointly by activations pertaining to individual pitches, transitions between pitches (separately for interval and contour), and the broader context defined by musical key (Figure 3). All components of sequence structure converge on nodes associated with serial position, which determines event sequencing. The assumption of shared representation accounts for AAF disruption by virtue of the interfering effect of perception on the activation of events for production. For instance, a serial shift adds activation 
to the contents of an event that had been performed earlier, and this may result in disruption of the pattern of activations within the representation across serial positions. Note that this architecture does not posit a reliance of actions on perceptual feedback and is therefore unlike feedback control theory. In the absence of auditory feedback, activations within the representation can still guide production.

\section{Dissociation of sequencing and timing representations}

The existing data suggest that not all time scales are treated similarly. In particular, the lower time scale of onset timing (related to perception/action synchrony), is dissociated from the higher time scale that involves sequential event order, at least with respect to the disruptive effects of AAF. It is not clear, either intuitively or given the data, that such a dissociation exists for different time scales that concern the serial order of events. (e.g., single pitches versus pitch transitions). It is possible that the dissociation between timing and sequencing results from the fact that the former is a continuous scale whereas the latter is best considered an ordinal scale.

Thus a model of the relationship between perception and action must address the apparent separation between the planning and perception of event onset times and the planning and perception of serial order. Node structure theory (MacKay, 1987) offers a possible way to conceptualize this distinction. In that theory, timing within the system is regulated by a set of nodes that is separate from those that regulate the retrieval or recognition of event contents. Biological oscillators that are sensitive to the complexity of phase relationships formed by emergent rhythms in a sequence may guide planning of timing (e.g., Jones, 1976; Kelso, 1995; Large \& Jones, 1999). By contrast, relationships between perception and action with respect to feedback contents may reflect disruption of memory retrieval (e.g., Palmer \& Pfordresher, 2003).

In the proposed framework, timing is considered to regulate activation levels for serial order nodes but not to influence associations between serial order and structural characteristics of sequence organization (pitch, contour, etc.). Timing thus determines when an event is produced, but not necessarily what event is produced. Figure 3 illustrates this point by showing change in activation across time within the level of the representation that is specific to timing. It is assumed that this activation function determines the activation of the current serial position, which in turn determines the activation of structural characteristics associated with the current position. However, event selection is determined by weights assigned to connections between nodes for serial order and structural characteristics.

\section{Components of perception/action similarity}

The current results imply that performers are sensitive to overall similarity between the planned sequence of pitches and the sequence of auditory feedback events. That is, it appears that disruption results when the feedback sequence is globally similar in structure, but presents a contrasting pattern of pitch motion relative to the planned event sequence. It is not hard to explain the disruptive effects of serial shifts, which could simply result because the node for an individual pitch that was performed earlier or is about to be performed is activated by perceptual input. Indeed, Node Structure Theory makes such a claim, but limits the time span over which such interference can occur (MacKay, 1987). However, it also appears that disruption is not limited to cases in which auditory feedback repeats past pitches or anticipates future ones.

It appears as though similarity based on higher time spans has a greater impact on relationships between perception and action than do relationships at lower time spans. When a perceived melody lacks the quality of diatonicity, no disruption results from serial shifts, even when the perceived melody shares its melodic contour with the planned melody (Pfordresher, 2006; cf. Dowling, 1978). Thus key seems to supersede contour. Furthermore, contour seems to supersede pitch. When feedback sequences are diatonic and share the same contour with the planned sequence but differ in pitch, serial shifting causes the same amount of disruption as does serial shifting of the actual planned sequence. However, feedback sequences that share the same set of pitches, but are dissimilar with respect to transitions between pitches yield less disruption (Pfordresher, 2005).

As a preliminary step in theory development, I computed correlations between structural components that may determine similarity and the overall amount of disruption that resulted from different feedback conditions when pitch was altered. Disruption for a given feedback condition and experiment was defined as the signed difference between error rates for altered and normal feedback conditions, divided by the error rate for normal feedback (similar to a Weber fraction). This measure of relative disruption correlated with different measures of similarity between the planned sequence and the feedback sequence. For the sake of brevity, I 


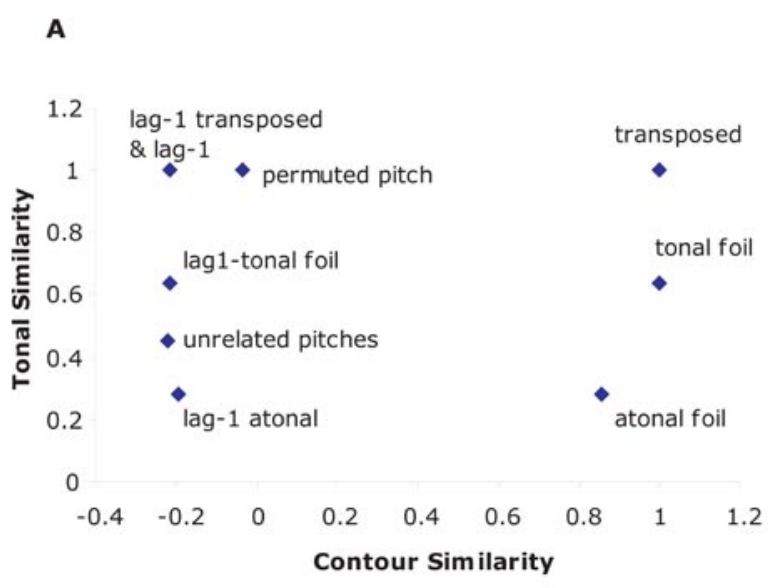

B

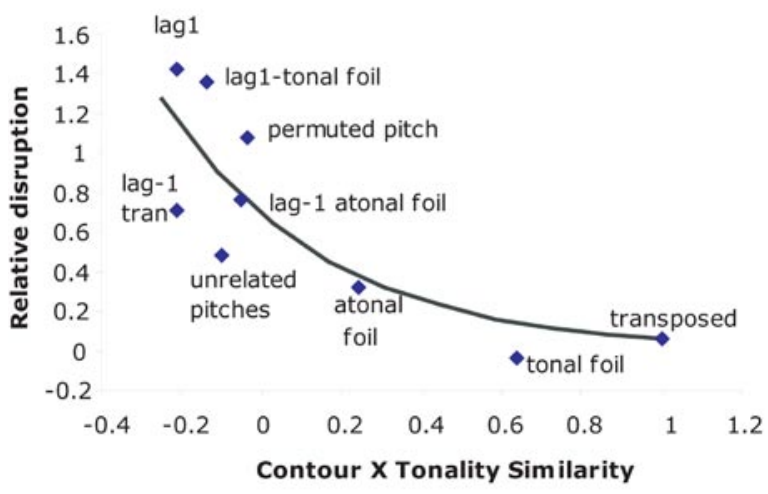

Figure 4.

(A) The relationship between two similarity metrics pertaining to altered-pitch feedback conditions. (B) The relationship between the interaction of these similarity metrics and disruption from different conditions. The solid line represents a linear least-squares fit of an exponential function. tran $=$ transposed.

focus here on the interaction between two theoretically motivated dimensions of similarity, melodic contour and tonality, which led to the most coherent prediction of relative disruption.

The relationship between these two dimensions is shown in Figure 4A. Similarity in contour was determined by correlating the pattern of pitch rises and falls in the feedback sequence with that of the planned sequence. These correlations predicted relative disruption $(r=-.80)$. Despite this strong correlation, contour similarity does not fully capture variation in disruption because groups of feedback conditions cluster around two points along the continuum (see abscissa of Figure 4A).

Tonal similarity was measured by correlating the feedback sequence and the planned sequence based on tone profiles (Krumhansl, 1990; Krumhansl \& Kessler, 1982). Specifically, the frequency of occurrence for pitch classes in each feedback sequence was correlated with the frequency distribution associated with the major diatonic scale (irrespective of key) found by Krumhansl \& Kessler (1983). Different fits were generated by treating each feedback pitch as a possible tonic pitch. Frequency distributions associated with best fits for each feedback sequence and produced sequences were correlated to gauge their relatedness with respect to the distribution of pitch classes. These correlations were weaker predictors of disruption than was contour similarity $(r=.25)$, but were independent of contour similarity $(r=-.08)$.

The interaction between melodic contour and tonal similarity, gauged by the product of both measures, proved to be an even better predictor of disruption than contour similarity alone (Figure 4B). Moreover, results reported earlier suggest that both contour and tonality influence disruption (Pfordresher, 2006). The relationship closely approximates an exponential function $\left(r^{2}=.92, r^{2}\right.$ for linear $\left.=.62\right)$, like similarity functions found in other research (e.g., Shepard, 1987). ${ }^{2}$ Consistent with the preliminary framework in Figure 3, disruption from altered contents thus reflects multidimensional similarity between planned and feedback sequences.

\section{The basis of "disruption" from AAF}

Researchers typically refer in a generic way to "disruption", with many changes in production serving as possible types of disruption. Such terminology is convenient shorthand, but theories will eventually need to determine the kinds of changes to the perception/action system that disrupt production. What, exactly, goes wrong? Two points have emerged from recent research.

First, disruption reflects the perturbation of perception/action coordination at a given time scale. This point emerges from the aforementioned dissociation between sequencing and timing (asynchronies disrupt timing and serial shifts disrupt accuracy). Thus, different kinds of disruption suggest that the perception/ action system treats sequential relationships across events as separate from onset synchronization.

Second, disruption may not simply reflect the passive response of the system to differences between planned and perceived events, but may also reflect active attempts to counteract the influence of auditory feedback. Current research suggests that responses to AAF reflect attempts to adapt planning during $A A F$, as discussed earlier. Thus, for instance, slowing of timing with asynchronous feedback may not simply reflect disruption per se (as proposed by Howell et al., 1983), but may reflect an attempt to position feedback onsets at phase locations that are less disruptive given 
the overall context (cf. Pfordresher \& Benitez, 2006; Pfordresher \& Palmer, 2002). Converging evidence for this claim stems from the finding that brain activity in temporo-parietal regions is enhanced when participants speak with DAF (Hashimoto \& Sakai, 2003). The authors interpreted these results as evidence for the use of "conscious self-monitoring...in addition to the automatic speech production system" (p. 26).

\section{The function of auditory feedback}

It is surprisingly easy to lose sight of an obvious theoretical question when researching the effects of AAF: What is the purpose of auditory feedback in the first place? According to the current data, oddly enough, auditory feedback does not function as "feedback" at all. In fact, were it not for the long history of the term I would be inclined to change it (as did Howell, 2004a, who changed it to "recurrent auditory information").

It seems that the use of auditory feedback, with respect to maintaining fluency in production, is limited to enhancing memory during learning (Finney \& Palmer, 2003), and possibly for monitoring fine nuances of performance (Repp, 1999). The results summarized here do not make a strong case for the utility of auditory feedback, but they do demonstrate the necessity of congruence between production and perception, perhaps reflecting a more general sensitivity to statistical regularities in the environment (cf. Saffran, 2003), including the relationships between actions and correlated perceptual events.

\section{Conclusions}

Recent findings concerning the effect of AAF suggest a characterization of how perception and action interact during music performance. In many respects, the nature of this interaction matches results from other domains. Motor control research, for instance, suggests that people use an "internal model" of the consequences of actions to help guide actions (e.g., Blakemore, Goodbody, \& Wolpert, 1998; Wolpert, Gharamani, \& Jordan, 1995), and other research cited earlier has similarly suggested that that perception and action share a common representation (Hommel et al., 2001; Mackay, 1987). Thus, the way in which people coordinate perception and action during music performance builds on a general tendency for people to relate action plans to perceptual information and may not rely on learned associations between movements and pitch events. This interpretation is supported by one of the more surprising findings of recent research, namely that the disruptive effect of AAF on keyboard performance is only moderately influenced by musical training.

\section{Acknowledgements}

This work was supported by NSF grant BCS$-0344892$.

\section{Notes}

${ }^{1}$ Serial shifts were initially referred to as period shifts to emphasize the distinction between phase and period (Pfordresher, 2003a).

2 The equation used was $\hat{Y}=a * e^{(X * b)}$. Best fitting parameter values (using linear least-squares optimization in MATLAB) were $a=0.69$ and $b=-2.47$.

\section{References}

Bangert, M., \& Altenmüller, E. O. (2003). Mapping perception to action in piano practice: A longitudinal DC-EEG study. BMC Neuroscience, 4, 26-40. Www

Benitez, B. (2005). Effects of Altered Auditory Feedback in Music Performance: The Role of Impulsivity, Musical Aptitude, and General Intelligence. Unpublished Master's Thesis, University of Texas at San Antonio, San Antonio, TX.

Bingham, G. P., Schmidt, R. C. \& Zaal, F. T. J. M. (1999). Visual perception of the relative phasing of human limb movements. Perception \& Psychophysics, 61, 246-258.

Black J. W. (1951). The effect of delayed side-tone upon vocal rate and intensity. Journal of Speech and Hearing Disorders, 16, 56-60. www

Blakemore, S. J., Goodbody, S. J., \& Wolpert, D. M. (1998). Predicting the consequences of our own actions: The role of sensorimotor context esimation. The Journal of Neuroscience, 18, 7511-7518.

Boltz, M. \& Jones, M.R. (1986). Does rule recursion make melodies easier to reproduce? If not, what does? Cognitive Psychology, 18, 389-431

Butler, R. A. \& Galloway, F. T. (1957). Factorial analysis of the delayed speech feedback phenomenon. Journal of the Acoustical Society of America, 29, 632-365.

Chase, R. A. (1965). An information-flow model of the organization of motor activity. I: Transduction, transmission and central control of sensory information. Journal of Nervous and Mental Disease, 140, 239-251.

Chase, R. A., Harvey, S., Standfast, S., Rapin, I. \& Sutton, S. (1961). Studies on sensory feedback I: Effect of delayed auditory feedback on speech and keytapping. Quarterly Journal of Experimental Psychology, 13, 141-152.

Cooper, G. \& Meyer L. B. (1960). The rhythmic struc- 
ture of music. Chicago : University of Chicago Press. Costa, P. T., Jr., \& McCrae, R. R. (1992). The Revised NEO Personality Inventory (NEO-PIR) and NEO FiveFactor Inventory (NEO-FFI) professional manual. Odessa, FL: Psychological Assessment Resources.

Costa-Giomi, E. (1999). The effects of three years of piano instruction on children's cognitive developement. Journal of Research in Music Education, 47, 198-212.

Costa-Giomi, E. (2004). Effects of three years of piano instruction on children's academic achievement, school performance, and self-esteem. Psychology of Music, 32, 139-152.

Dell, G. S. (1986). A spreading-activation theory of retrieval in sentence production. Psychological Review, 93, 283-321. WwW

Dowling, W. J. (1978). Scale and contour: Two components of a theory of memory for melodies. Psychological Review, 85, 341-354.

Drost, U. C., Rieger, M., Brass, M., Gunter, T. C., \& Prinz, W. (2005). Action-effect coupling in pianists. Psychological Research, 69, 233-241. |www

Fairbanks, G. \& Guttman, N. (1958). Effects of delayed auditory feedback upon articulation. Journal of Speech and Hearing Research, 1, 333-346. |www

Finney, S. A. (1997). Auditory feedback and musical keyboard performance. Music Perception, 15, 153$-174$.

Finney, S. A. (1999). Disruptive Effects of Delayed Auditory Feedback on Motor Sequencing. Unpublished doctoral dissertation, Brown University, Providence, RI.

Finney, S. A. (2001). FTAP: A Linux-based program for tapping and music experiments. Behavior Research Methods, Instruments \& Computers, 33, 65-72. WWw

Finney, S. A. \& Palmer, C. (2003). Auditory feedback and memory for music performance: Sound evidence for an encoding effect. Memory \& Cognition, 31, 51-64.

Finney, S. A., \& Warren, W. H. (2002). Delayed auditory feedback and rhythmic tapping: Evidence for a critical interval shift. Perception \& Psychophysics, 64, 896-908. $\mid \underline{w w \mid}$

Fromkin, V. A. (1971). The nonanomalous nature of anomalous utterances. Language, 47, 27-52.

Gabrielsson, A. (1999). The performance of music. In D. Deutsch (Ed.) The Psychology of Music $2^{\text {nd }}$ Ed. (pp. 501-602). San Diego : Academic Press.

Gabrielsson, A. (2003). Music performance research at the millenium. Psychology of Music, 31, 221-272.

Garrett, M. F. (1980). Levels of processing in sentence production. In B. Butterworth (Ed.), Language pro- duction: Speech and talk (pp. 177-220). London: Academic Press.

Gates, A., \& Bradshaw, J. L. (1974). Effects of auditory feedback on a musical performance task. Perception \& Psychophysics, 16, 105-109.

Gates, A., Bradshaw, J., \& Nettleton, N. (1974). Effect of different delayed auditory feedback intervals on a music performance task. Perception \& Psychophysics, 14, 21-25.

Greenwald, A. G. (1970). Sensory feedback mechanisms in performance control: With special reference to the ideo-motor mechanism. Psychological Review, 77, 73-99. WWW

Hashimoto, Y., \& Sakai, K. L. (2003). Brain activations during conscious self-monitoring of speech production with delayed auditory feedback: An fMRI study. Human Brain Mapping, 20, 22-28. |WwW

Havlicek, L. (1968). Effects of delayed auditory feedback on musical performance. Journal of Research in Music Education, 16, 308-318.

Hommel, B., Müsseler, J., Aschersleben, G. \& Prinz, W. (2001). The theory of event coding (TEC): A framework for perception and action planning. Behavioral and Brain Sciences, 24, 849-937. WWW

Houde, J. F., \& Jordan, M. I. (1998). Sensorimotor adaptation in speech production. Science, 279, 1213-1216. WWW

Howell, P. (2001). A model of timing interference to speech control in normal and altered listening conditions applied to treatment of stuttering. In B. Massen, W. Julstijn, R. Kent. H. F. M. Peters. \& P. H. M. M. Van Lieshout (Eds.) Speech Motor Control in Normal and Disordered speech (pp. 291-294). Nijmegen : Uttgeverij Vantilt.

Howell, P. (2004a). Effects of delayed auditory feedback and frequency-shifted feedback on speech control and some potentials for future development of prosthetic aids for stammering. Stammering Research, 1, 34-43.

Howell, P. (2004b). Assessment of some contemporary theories of stuttering that apply to spontaneous speech. Contemporary Issues in Communicative Sciences and Disorders, 39, 122-139.

Howell, P. \& Archer, A. (1984). Susceptibility to the effects of delayed auditory feedback. Perception \& Psychophysics, 36, 296-302. |WWw

Howell, P., Powell, D. J., \& Khan, I. (1983). Amplitude contour of the delayed signal and interference in delayed auditory feedback tasks. Journal of Experimental Psychology: Human Perception \& Performance, 9, 772-784.

Ivry, R., Diedrichsen, J., Spencer, R., Hazeltin, E., \& 
Semjen, A. (2004). A cognitive neuroscience perspective on bimanual coordination and interferences. In S. Swinnen \& J. Duysens (Eds.). Neuro-Behavioral Determinants of Interlimb Coordination (pp. 259-295). Boston : Kluwer Academic Publishing.

James. W. (1890). Principles of psychology (volume 2). New York : Holt.

Jones, M. R. (1976). Time, our lost dimension: Toward a new theory of perception, attention, and memory. Psychological Review, 83, 323-355. WWW

Jones, M. R. (1987). Dynamic pattern structure in music: Recent theory and research. Perception \& Psychophysics, 41, 621-634. WWW

Jones, M. R., \& Boltz, M. (1989). Dynamic attending and responses to time. Psychological Review, 96, 459-491. Www

Jones, M. R. \& Holleran, S. (1992). Cognitive bases of musical communication. Washington, DC : American Psychological Association.

Jones, M. R. \& Pfordresher, P.Q. (1997). Tracking melodic events using joint accent structure. Canadian Journal of Experimental Psychology, 51, 271-291.

Juslin, P. N. \& Sloboda. J. A. (2001). Music and emotion: Theory and research. Oxford : Oxford University Press.

Kalmus, H. Denes, F., \& Fry, D. B. (1955). Effect of delayed acoustic feedback on some nonvocal activities. Nature, 175, 1078.

Kelso, J. A. S. (1995). Dynamic Patterns. Cambridge, MA : MIT Press.

Krampe, R. T., Mayr,U., \& Kliegl, R. (2005). Timing, sequencing, and executive control in repetitive movement production. Journal of Experimental Psychology: Human Perception and Performance, 31, 379-397. Www

Krumhansl, C. L. (1990). Cognitive Foundations of Musical Pitch. Oxford : Oxford University Press.

Krumhansl, C. L., \& Kessler, E. J. (1982). Tracing the dynamic changes in perceived tonal organization in a spatial representation of muscal keys. Psychological Review, 89, 334-368. $\mid \underline{W W \mid}$

Krumhansl, C. L. \& Shepard, R. N. (1979). Quantification of the hierarchy of tonal functions within a diatonic context. Journal of Experimental Psychology: Human Perception and Performance, 5, 579-594. www

Large, E. W. \& Jones, M. R. (1999). The dynamics of attending: How people track time-varying events. Psychological Review, 106, 119-159.

Lashley, K. (1951). The problem of serial order in behavior. In L. A. Jeffress (Ed.) Cerebral mechanisms in behavior (pp. 112-136). New York : Wiley.

Lehiste, I. (1977). Isochrony reconsidered. Journal of
Phonteics, 5, 253-263.

Levelt, W. J. M. (1989). Speaking: From intention to articulation. Cambridge, MA : MIT Press.

Lerdahl, F. \& Jackendoff, R. (1983). A Generative Theory of Tonal Music. Cambridge, MA : MIT Press.

Lee, B. S. (1950). Effects of delayed speech feedback. Journal of the Acoustical Society of America, 22, 824-826.

Lee, B. S. (1951). Artificial Stutter. Journal of Speech and Hearing Disorders, 16, 53-55.

MacKay, D. G. (1968). Metamorphosis of a critical interval: Age-linked changes in the delay in auditory feedback that produces maximal disruption of speech. Journal of the Acoustical Society of America, 43, 811-821.

MacKay, D. G. (1987). The organization of perception and action. New York: Springer-Verlag.

Müsseler, J. (1999). How independent from action control is perception? An event coding account for more equally-ranked crosstalks. In G. Aschersleben, T. Bachmann, \& J. Müsseler (Eds.) Cognitive Contributions to the perception of spatial and temporal events (pp. 121-148). Amsterdam : Elsevier.

Palmer, C. (1997). Music performance. Annual Review of Psychology, 48, 115-138. | |ww|

Palmer, C., \& Drake, C. (1997). Monitoring and planning capacities in the acquisition of music performance skills. Canadian Journal of Experimental Psychology, 51, 369-384. |WWw

Palmer, C., \& Krumhansl, C. L. (1990). Mental representations for musical meter. Journal of Experimental Psychology: Human Perception and Performance, 16, 728-741. $\mid \underline{w W \mid}$

Palmer, C. \& Pfordresher, P.Q. (2003). Incremental planning in sequence production. Psychological Review, 110, 683-712. $\frac{|w w|}{w w}$

Peretz, I., Champod, S., \& Hyde, K. (2003). Varieties of musical disorders: the Montreal Battery of Evaluation of Amusia. Annals New York Academy of Sciences, 999, 58-75. Www

Pfordresher, P.Q. (2003a). Auditory feedback in music performance: Evidence for a dissociation of sequencing and timing. Journal of Experimental Psychology: Human Perception and Performance, 29, 949-964. Www Pfordresher, P. Q. (2003b). The role of melodic and rhythmic accents in musical structure. Music Perception, 20, 431-464.

Pfordresher, P. Q. (2005). Auditory feedback in music performance: The role of melodic structure and musical skill. Journal of Experimental Psychology: Human Perception and Performance. Www

Pfordresher, P. Q. (2006). Relationships between move- 
ment trajectories and pitch trajectories in music performance as a basis for perception/action similarity. Manuscript in preparation.

Pfordresher, P. Q. \& Benitez, B. (2006). Temporal coordination of actions and sound during sequence production. Manuscript in preparation.

Pfordresher, P. Q. \& Palmer, C. (2002). Effects of delayed auditory feedback on timing of music performance. Psychological Research, 66, 71-79. WWW

Pfordresher, P. Q. \& Palmer C. (in press). Effects of hearing the past, present, or future during music performance. Perception \& Psychophysics. WWW

Piedmont, R. L. (1998). The Revised NEO Personality Inventory: Clinical and Research Applications. New York : Plenum Press.

Prinz, W. (1997). Perception and action planning. European Journal of Cognitive Psychology, 9, 129$-154$.

Rauscher, F., Shaw, G., Levine, L., Wright, E., Dennis, W., \& Newcomb, R. (1997). Music training causes long-term enhancement of preschool children's spatial-temporal reasoning. Neurological Research, 19, 2-8. WWw

Repp, B. H. (1999). Effects of auditory feedback deprivation on expressive piano performance. Music Perception, 16, 409-438.

Repp, B. H., \& Knoblich, G. (2004). Perceiving action identity: How pianists recognize their own performance. Psychological Science, 15, 604-609. WWW

Rizzolatti, G., Fogassi, L., \& Gallese, V. (2001). Neurophysiological mechanisms underlying the understanding and imitation of action. Nature Reviews Neuroscience, 2, 661-670. $\underline{\mathrm{ww}}$

Robinson, G. M. (1972). The delayed auditory feedback effect is a function of speech rate. Journal of Experimental Psychology, 95, 1-5. Www

Rosenbaum, D. A. (1991). Human motor control. San Diego, CA : Academic Press.

Saffran, J. R. (2003). Statistical language learning:
Mechanisms and constraints. Trends in Cognitive Science, 4, 110-114.

Schellenberg, E. G. (2004). Music lessons enhance IQ. Psychological Science, 15, 511-514. |WwW

Schmidt, R. A. \& Lee, T. D. (1999). Motor control and learning: A behavioral emphasis. Champaign, IL : Human Kinetics.

Shepard, R. N. (1987). Toward a universal law of generalization for psychological science. Science, 237, 1317-1323.

Singer, W. (1993). Synchronization of cortical activity and its putative role in information processing and learning. Annual Review of Physiology, 55, 349-374. WWW

Sloboda, J. A. (1982). Music performance. In D. Deutsch (Ed.) Psychology of Music. New York : Academic Press.

Sloboda, J. A. (1985). The Musical Mind. Oxford : Oxford University Press.

Sloboda, J. A. (2005). Exploring the Musical Mind. Oxford : Oxford University Press.

Smith, K. U. (1962). Delayed Sensory Feedback and Behavior. Philadelphia : W. B. Saunders Company.

Thomassen, J. M. (1982). Melodic Accent: Experiments and a tentative model. Journal of the Acoustical Society of America, 71, 1596-1605. Www

Turvey, M. T. (1990). Coordination. American Psychologist, 48, 938-953. [WwW

Wolpert, D. M., Ghahramani, Z., \& Jordan, M. I. (1995). An internal model for sensorimotor integration. Science, 269, 1880-1882. |www

Wonderlic, E. (1992). Wonderlic personnel test. Wonderlic, Inc. : Libertyville, IL.

Yates, A. J. (1963). Delayed auditory feedback. Psychological Bulletin, 60, 213-232. |WWW

Zaal, T. J., Bingham, G. P. \& Schmidt, R. C. (2000). Visual perception of mean relative phase and phase variability. Journal of Experimental Psychology: Human Perception and Performance, 26, 1209-1220. www 